\title{
Principles of the non-Euclidian model application to the problem of dissipative mesocracking structures of highly compressed rock and massifs modelling
}

\author{
Mikhail Guzev" and Vladimir Makarov ${ }^{1}$ \\ ${ }^{1}$ Far-Eastern Federal University, Vladivostok, Primorskiy krai, Russia.
}

\begin{abstract}
New experimental results such as "zonal disintegration" around deep openings and "reversible deformations" of highly compressed rock samples cannot be described correctly from contemporary rock mechanics, which is based on the principals of classical Continuum Mechanics theory. A new approach to rock mechanics mathematical models consists of the application of non-Euclidian modelling to the problem of the description of anomalous experimental results. This leads to the formation of the "Geomechanics of Highly Compressed Rock and Rock Massifs" - a new branch of the existing theory of Geomechanics - in which framework a radical rise in geodynamical phenomena forecasting can be achieved. Principles of the geomechanics of highly compressed rock and rock massifs are discussed in this paper. The effectiveness of the application of non-Euclidian modelling to the anomalous experimental effects observed in research is demonstrated on two hierarchical geomedia block levels such as rock samples and rock massif around underground openings. Keywords: reversible deformation, zonal disintegration, non-Euclidian modelling, high compression.
\end{abstract}

\section{Introduction}

Research on the deformation and failure relationships of rock massifs, which are in conditions of high compression - exceeding the strength by one half - has shown two noteworthy experimentally determined abnormal phenomena: the reversible deformation of rock samples [1-3] and the zonal character of the deformation and failure of the rock massif around underground openings [4, 5] (Fig. 1).

It is impossible to explain both of these new phenomena from the standpoint of existing classical representations of geomechanics. Attempts at providing a theoretical description of these separate effects, undertaken by a number of authors, have not led to success [6-10]. A general theory which would allow us to explain all of the available experimental data from a uniform position does not currently exist.

At the same time, representations in other areas of science regarding thermodynamically nonequilibrium processes have been developed for open energy systems and demanding the

\footnotetext{
*Corresponding author:vlmvv@mail.ru
} 
statement of the local irreversibles [11]. Gauge theory methods of representing a defective continuum have been applied to the mechanics of continuous media, allowing a transition to a new class of models in which the defective media has been replaced by an equivalent continuous one via the introduction of a non-Euclidian (curved) model space [12].

Localization of the defect appearance processes, in turn, has led to the mesomechanics representation in which attention has been focussed on the processes of formation and development of the various types mesostructures in a continuum media with defects, which are the areas prepared for the occurrence of macrodefects [13-15]. Moreover, a rock massif under the conditions found at great depths can be represented as an open nonequilibrium thermodynamics system of defective media $[16,17]$. All of the positions are complicated by the hierarchical character of defects existing in a rock massif according to its hierarchical block structure [18].

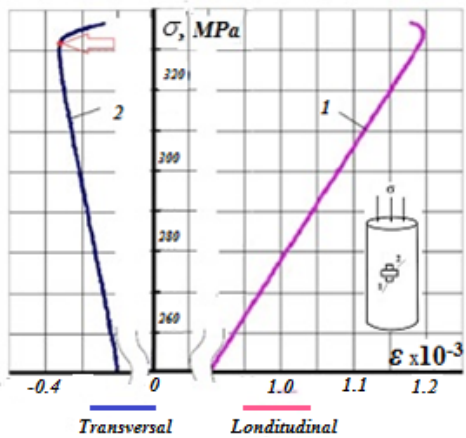

a

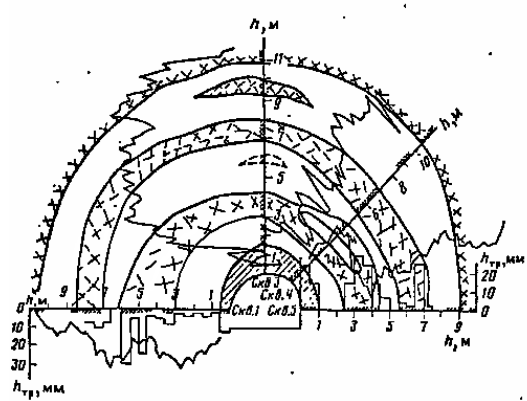

b

Fig. 1. Anomalous phenomena of highly compressed rock massifs: a - Reversible deformation of rock samples [3], b - Zonal character of destruction near deep underground openings [4].

Therefore, the development of a new model of a rock massif, which is under the conditions found at great depths, has demanded the creation of a theoretical base corresponding to the new requirements, discovering new, abnormal from the point of view of the existing theory, the phenomena of deformation and destruction of rock.

\section{Non-Euclidian model to simulate anomalous geomedia phenomena}

Our approach uses a non-Euclidian model of the zonal failure phenomena description on the hierarchical level of the mining opening scale of the geomedia block [19]. The model describing the zonal structure of the stress field around a cylindrical opening is considered in a stationary formulation and is assumed to be a plane loosed by a circular hole. For the non-Euclidean model, the classical equations of equilibrium and the boundary conditions remain true:

$$
\frac{\partial \sigma_{i j}}{\partial x_{j}}=0 .
$$

In this model, the stress $\sigma_{\infty}$ is specified at infinity under the condition that $\sigma_{r}=0$ at the opening boundary $r=r_{0}$, where $r_{0}$ is radius of the opening. In accord with the linearity of equation (1), it is possible to present the required solution as the sum of the classical $\sum_{i j}$ field and an additional field $T_{i j}$ :

$$
\sigma_{i j}=\Sigma_{i j}+T_{i j} .
$$


The $T_{i j}$ field is reflected by the parameter of incompatibility $R$. In a plane-strain approximation, for deep openings with a circular cross-section, we have a $R$-function

$$
\frac{R}{2}=2 \frac{\partial^{2} \varepsilon_{12}}{\partial x^{1} \partial x^{2}}-\frac{\partial^{2} \varepsilon_{11}}{\partial x^{2} \partial x^{2}}-\frac{\partial^{2} \varepsilon_{22}}{\partial x^{1} \partial x^{1}},
$$

that characterizes the incompatibility of the components $\varepsilon_{i j}$. In polar coordinates, we have the following expression for the stress components [19]:

$$
\sigma_{r}=\sigma_{\infty}\left(1-\frac{r_{0}^{2}}{r^{2}}\right)+(\gamma+\beta-\alpha) \frac{\partial^{2} R}{\partial r^{2}}+\frac{\gamma}{r} \frac{\partial R}{\partial r}, \sigma_{\theta}=\sigma_{\infty}\left(1+\frac{r_{0}^{2}}{r^{2}}\right)+(\gamma+\beta) \frac{\partial^{2} R}{\partial r^{2}}+\frac{\gamma}{r} \frac{\partial R}{\partial r},
$$

where $\alpha, \beta, \gamma$ are constant coefficients.

This model (1) - (4) can describe the periodical character of the stress field around a cylindrical opening. The results of the boundary problem solution give the formulas for the stress components:

$$
\begin{aligned}
& \sigma_{r r}=\sigma_{\infty}\left(1-\frac{r_{0}^{2}}{r^{2}}\right)-\frac{E}{2\left(1-v^{2}\right) \gamma^{3 / 2}} \cdot \frac{1}{r} \times\left[a J_{1}(\sqrt{\gamma r})+b N_{1}(\sqrt{\gamma r})+c K_{1}(\sqrt{\gamma r})\right] \\
& \sigma_{\varphi \varphi}=\sigma_{\infty}\left(1+\frac{r_{0}^{2}}{r^{2}}\right)-\frac{E}{2\left(1-v^{2}\right) \gamma} \times\left[a J_{0}(\sqrt{\gamma} \cdot r)+b N_{0}(\sqrt{\gamma} \cdot r)-c K_{0}(\sqrt{\gamma} \cdot r)\right]+ \\
& +\frac{E}{2\left(1-v^{2}\right) \gamma^{3 / 2}} \cdot \frac{1}{r}\left[a J_{1}(\sqrt{\gamma} \cdot r)+b N_{1}(\sqrt{\gamma} \cdot r)+c K_{1}(\sqrt{\gamma} \cdot r)\right],
\end{aligned}
$$

where $r$ is the radius-vector, which characterizes the zones positions.

After carrying out the calculations with the failure criteria [20], it was determined that the characteristics of the zones depend slightly on the values of the deformational parameters of the model: E, the elastic modulus of the rock, and $v$, the Poisson ratio. The effectiveness of the model has been demonstrated using wide cases of rock massif properties for solid rock $\left(\sigma_{c}=150 \mathrm{MPa}\right.$, where $\sigma_{c}$ is rock strength) and for weak rock $\left(\sigma_{c}=\right.$ $15 \mathrm{MPa}$ ). Algorithms and programs have been developed to calculate the expressions of the stresses, and the criterion function $K(r)$ [5].

Here, the results of the analysis of zonal failure in the Nikolaevskij ore mine (Dalnegorsk, Russia) are presented. The forecasted depths of the failure zones are shown in Table 1. The correlation between the theory and the experimental research has been estimated by comparing the results of in situ measurements of the radial displacements near the openings (Nikolaevskij ore mine) with the model predictions (Fig. 2). It was determined that the quantitative difference between the forecasted and measured data was no more than $47 \%$, and the full quality satisfaction.

A comparison of the analytical and experimental results for soft rock also shows good correspondence. The rock analyses can be summarized by stating that the basic factor influencing the zonal failure structure parameters is the value of the stress level in the rock massif. With increasing depth, the number of failure zones increases and their radial width also increases.

The anomalous character of the strains in rock samples (Fig. 1a) can also be described by the non-Euclidian model [21]. Extensive natural and experimental studies carried out in laboratories worldwide allow us to make conclusions about the inhomogeneous space-time allocation of longitudinal and lateral deformations of local fields of the sample (Fig. 3). 

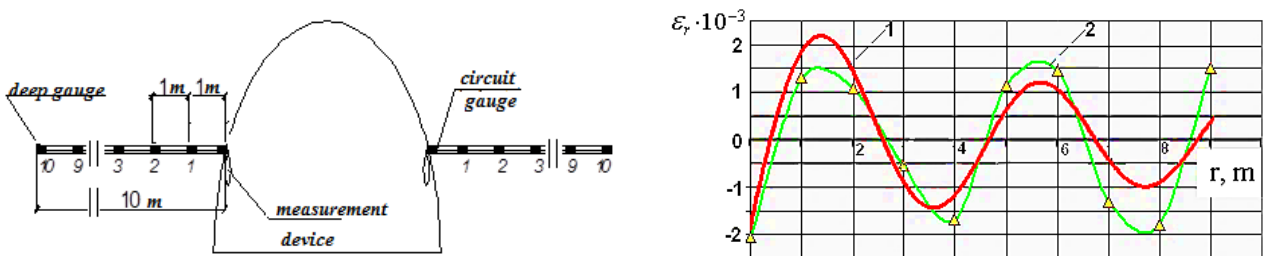

Fig. 2. Theoretical (1) and experimental (2) radial deformations near the opening (right) and research station (left)

Table 1. Depth of zonal structure forecasting

\begin{tabular}{|c|c|c|c|c|}
\hline Zone Destruction Number & I & II & III & IV \\
\hline Critical relative stress of zone formation & 1.3 & 2.3 & 2.9 & 3.3 \\
\hline Depth of zone appearance (m) & 520 & 920 & 1160 & 1320 \\
\hline
\end{tabular}

The phenomenon of the localisation of macroscopic deformations is the motivating force behind the further application of the non-Euclidian model to the study of rock sample behaviour allowing for a dilation of the possibilities for the theoretical description of the rock massif medium.

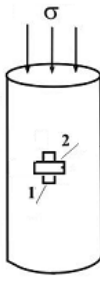

a

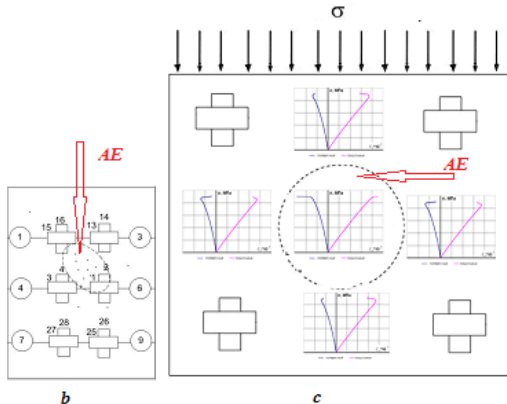

Fig. 3. Mesocracking structure of the source type:

$\mathrm{a}$-Scheme of loading (1, 2 - linear deformation gauges), $\mathrm{b}$ - Source area location (1-9-AE sensors), $\mathrm{c}$ - Reversible deformation location in the near-source area (AE - acoustic emission)

The boundary problem for the allocation of a field of stresses $\sigma_{i j}$ in a cylindrical sample is formulated in a stationary statement (1). The solution is written in the form (1). Let the external force $\boldsymbol{P}=(0,0, P)$ act on the sample ends. Then for balance it is compensated by the force of internal stresses, and the boundary conditions are satisfied at the ends of the sample $\left(S_{ \pm}\right)$and on its lateral surface $(\Gamma)$ :

$$
\int_{S_{ \pm}} \sigma_{i j} n_{j} d S=0, \int_{\Gamma} \sigma_{z j} n_{j} d S=\int_{\Gamma} P d S .
$$

The demands (5) are satisfied on the condition that

$$
\begin{gathered}
\left.\sum_{z z}\right|_{z= \pm h}=P,\left.\quad \sum_{z r}\right|_{z= \pm h}=0,\left.\quad \sum_{z \varphi}\right|_{z= \pm h}=0, \\
\left.\sum_{r r}\right|_{r=R}=0,\left.\quad \sum_{r \varphi}\right|_{r=R}=0,\left.\quad \sum_{r z}\right|_{r=R}=0 \\
\left.\int_{0}^{R} r d r \int_{0}^{2 \pi} d \varphi T_{i z}\right|_{z= \pm h}=0,\left.\quad \int_{-h}^{h} d z \int_{0}^{2 \pi} d \varphi T_{i r}\right|_{r=R}=0,
\end{gathered}
$$

where $R$ is the radius of the sample and $2 h$ is its height. The conditions (6) coincide with the classical conditions locally at each point. It is seen from (7) that the components $T_{i j}$ are the self-balanced fields. 
To construct $T_{i j}$ the stress function $g$ must be introduced:

$$
T_{i j}=\delta_{i j} \Delta g-\frac{\partial^{2} g}{\partial x^{i} \partial x^{j}} .
$$

It is well known that the classical theory demands $\Delta \Delta g=0$, where $\Delta$ is the Laplace operator. Then $T_{i j}$ satisfies the compatibility conditions. The non-Euclidian model results in the equation

$$
\Delta g=-\gamma g .
$$

with a phenomenological parameter $\gamma$. In the cylindrical coordinate system, we have the following expression for the stress components (8):

$$
\begin{aligned}
& T_{r r}=\frac{1}{r} \frac{\partial g}{\partial r}+\frac{1}{r^{2}} \frac{\partial^{2} g}{\partial \varphi^{2}}+\frac{\partial^{2} g}{\partial z^{2}}, \quad T_{r \varphi}=-\frac{\partial}{\partial r}\left(\frac{1}{r} \frac{\partial g}{\partial \varphi}\right), T_{r z}=-\frac{\partial}{\partial r} \frac{\partial g}{\partial z}, \\
& T_{\varphi \varphi}=\frac{\partial^{2} g}{\partial r^{2}}, T_{z \varphi}=-\frac{\partial}{\partial z}\left(\frac{1}{r} \frac{\partial g}{\partial \varphi}\right), T_{z z}=\frac{\partial^{2} g}{\partial r^{2}}+\frac{1}{r} \frac{\partial g}{\partial r}+\frac{1}{r^{2}} \frac{\partial^{2} g}{\partial \varphi^{2}} .
\end{aligned}
$$

The common solution of the field components $T_{i j}$ is expressed through a Fourier series. The model numerical calculation was carried out for the experimental research using rock sample characteristics: $v=0.26, \mathrm{E}=1.7 * 10^{4} \mathrm{MPa}, \mathrm{h}=5 \mathrm{~cm}, \mathrm{R}=2.5 \mathrm{~cm}$. The calculation results for the deformations at the same experimental conditions show a full qualitative coincidence of the analytical and experimental results, and the maximum quantitative difference of the strain values does not exceed $23 \%$ [21].

\section{Geomechanics of highly compressed rock massifs: subject and method.}

The novelty of the theory is provided by the subject of the research, which does not have an analogue in existing theoretical conceptions. Additionally, the new theory demands, besides new conceptual definitions, the introduction of our own method of research.

The subject of the Geomechanics of Highly Compressed Rock and Rock Massifs is the rock objects, which hierarchical block massifs generate at each level of the geomedia, and the similar wave jointing mesostructures of the contrast type, which are areas of preparation for macrodefects of the following hierarchical level.

The method of the Geomechanics of Highly Compressed Rock and Rock Massifs consists of the representation of hierarchical block geomedia by a system of non-Euclidian models and the replacement of mesodefective structures in each hierarchical level of the defective media by a model of the continuous media in which the presence of defects is replaced with a condition of curvature of the model space (a condition of incompatibility) $[21,22]$, and the occurrence of macrodefects is defined by a system of strength criteria [20]. In this case, the macrodefects of the bottom hierarchical level are mesodefects of the neighbouring upper hierarchical level of the geomedia.

\section{Principles of the Geomechanics of Highly Compressed Rock and Rock Massifs}

The basic consequences of the experimental data analyses and the non-Euclidian approach to modelling may be formulated as the following scientific positions. The rock mass represents hierarchical block medium with crystal-like formations (minerals) located at the 
lowest level, and the highest level is limited to the scale of the objects considered within the crust. Dissipative mesocrack structure (DMS) is considered as a set of areas of active (increase) and passive (decrease) defect formation. At each hierarchical level, it is reasonable to consider three scale levels of defects, i.e. micro- meso- and macrodefects, which makes difficult the analysis of such media and construction of adequate mathematical models. Macrodefects of the lowest hierarchical level can be considered mesodefects at the next higher hierarchical level (macro-meso-transition).

Conception of "high compression of rocks and rock mass" characterizes formation and development of the dissipative mesocrack structures (DMS) of the corresponding hierarchical level. So, subject of the geomechanics of highly compressed rocks and rock mass is the rock phenomena generated by hierarchical rock mass at each level of geomedium, Method of geomechanics of 'highly compressed rocks and rock mass' is presentation of hierarchical block geomedium by a system of non-Euclidian models. Thus, we replace mesodefective structures at each hierarchical level of the defective medium by the models of the continuous medium. Therefore, main principle of geomechanics of highly compressed rock and rock mass is the principle of non-Euclidian hierarchy of geomedium, i.e. replacement of block hierarchical medium by continuous hierarchically structured medium with the non-Euclidian metrics, where transition between structural levels (models) is defined by condition criteria corresponding to the meso-macro-meso process. The conditions of compatibility of elastic deformations are not satisfied in each point in the defective medium, while conditions of compatibility of general deformations for the corresponding hierarchical level of blocks remain satisfied (the monolithic block principle).

\section{Some Conditions of Geomechanics of Highly Compressed Rock and Rock Massifs applicability}

Some conditions of the geomechanics of highly compressed rocks and rock mass applicability must be added to the principles described above. These are the condition of the geomedium being far from thermodynamic balance, the condition of transference from one structural level of the hierarchical block medium to another, and the condition of criteria satisfaction [21-22].

\section{Formation of a new scientific branch of Geomechanics}

Representations regarding the reliability of the forecast of geodynamic phenomena are based on reliable precursors of the formation and development stages of the source type mesocracking structures in a massif of the corresponding hierarchical level [25]. The formation of zonal type structures around deep mining openings (zone disintegration) forces us to reconsider the principles describing the interaction of the lining with a rock massif and the technology employed in its construction [26].

\section{Conclusion}

The Geomechanics of Highly Compressed Rock and Rock Massifs is a new scientific branch of general Geomechanics in which, on the basis of considering a rock massif under the conditions found at great depths from the standpoint of nonequilibrium thermodynamics and gauge theories of the defective media, models describing mesocracking structures at all levels of the block hierarchy are developed, with the designation of a method of transitioning from the mesostructure of each lower level to similar structures of upper levels through the corresponding meso-macro process. 


\section{Acknowledgements}

The authors thank Prof. V. Odintsev for his important recommendations in the process of writing this paper. We thanks moreover PhD L. Ksendzenko for her income to the models calculation and $\mathrm{PhD}$ A. Golosov for the experimental research results. The research is supported by a grant from the Ministry of Education and Science of the Russian Federation No. RFMEFI58418X0034.

\section{References}

1. Seldenrath T. R., Gramberg J. Mech. Prop. of Non-Metallic Materials, Butterworths, pp. 79-102. (1958)

2. Guzev M. A., Makarov V. V. Deformability and failure of highly stressed rocks around openings. 231 P. (in Russian) (2007)

3. Guzev M. A., Makarov V. V., Ushakov A. A. J. Mining Sci., 6. 3-13. (2005)

4. Shemyakin, E. I., Fisenko, G. L., et al. Journal of Mining Science. 3. 3-15. (1986)

5. Vladimir, V., Makarov, M., A. Guzev, V. N. Odintsev, L. S. Ksendzenko. Journal of Rock Mechanics and Geotechnical Engineering. 8 (2). 164-169. (2016)

6. Shemyakin, E. I., Fisenko, G. L., Kurlenya, M. V., Oparin, V. N., et al. Journal of Mining Science. 1. 3-8. (1987)

7. Shemyakin, E. I., Fisenko, G. L., Kurlenya, M. V., Oparin, V. N., et al. Proc. of the USSR Academy of Sciences. 289 (5). 1088-1094. (1986)

8. Qian, Q. H., Zhou, X. P., Yang, H. Q., Zhang, Y. X., Li, X. H. Theoretical and Applied Fracture Mechanics. 51 (2). 129-138. (2009)

9. Reva, V. N., Tropp, E. A. Proceedings of Physics and the Mechanics of Rocks Failure, VNIMI Publishing: Saint Petersburg, pp. 125-130. (in Russian) (1995)

10. Chanyshev, A. I. Intense-deformed condition of a rock massif, Novosibirsk: Mining Inst. pp. 3-8 (in Russian) (1988)

11. Prigogine, I., Kondepudi, D. Modern thermodynamics. Pub. 1998 by John Wiley \& Sons Ltd, 506 P. (1998)

12. Kadich, A., Edelen, D. The gauge theory of dislocation and disclination, M: World, 168 P. (in Russian) (1987)

13. Panin, V. E., Grinjaev, J. V., et al. Structural levels of plastic deformation and destruction, Novosibirsk: Science, 255 P. (in Russian) (1990)

14. Panin, V. E., ed. The physical mesomechanics and computer designing of materials: in 2 v., Novosibirsk: Science, 1, 297 P.; 2, 320 P. (in Russian) (1995)

15. Lockner, D. A., Byerlee, J. D., Kuksenko V., Ponomarev A., Sidorin A. Nature. 350 (7). 39-42. (1991)

16. Guzev M. A., \& Myasnikov V. P. Mech. solids, 33(4), 131-144 (in Rus) (1998)

17. De Grotto, C., Masur, P. Nonequilibrium thermodynamics. World, 524 P. (in Russian) (1964)

18. Sadovski, M. A. Natural blocky of rock, Proc. AS USSR. 247 (4). (in Rus) (1979)

19.Guzev M. A. J. Rock Mechanics and Geotechnical Engineer. 6(3). 180-185. (2014)

20. Odintsev, V. N. Tension destruction of a massif of brittle rocks. IPKON, RAS, 166 P. (in Russian) (1996)

21. Guzev M., Makarov V., Ksendzenko L. ISRM Regional Symposium "EUROCK 2015: Future Development of Rock Mechanics", EUROCK 2015 \& 64th Geomechanics Colloquium, Salzburg, Austria. pp. 979-984. (2015)

22. Goldin, S. V. Physical Mesomechanics, 8 (1). 5-14. (in Russian) (2005)

23. Guzev, M. A., Odintsev, V. N., \& Makarov, V. V. Tunnelling and Underground Space Technology, 81, 506-511 (2018). https://doi.org/10.1016/j.tust.2018.08.018. 